\title{
Heavy quark probes of QCD
}

\author{
Nuno Leonardo* \\ LIP and University of Lisbon \\ E-mail: nuno.leonardo@cern.ch \\ on behalf of the CMS Collaboration
}

Large heavy flavor enriched data sets are being accumulated by the CMS experiment in different collision environments and energies provided by the LHC. These offer robust probes for testing the standard model and potentially reaching beyond it. In this report I concentrate on recent production measurements in proton-proton, ion-ion and proton-ion collisions. These allow to probe the underlying QCD mechanisms of hadron formation and the properties of QCD matter under extreme temperatures.

The 39th International Conference on High Energy Physics (ICHEP2018)

4-11 July, 2018

Seoul, Korea

${ }^{*}$ Speaker. 
CMS is a general purpose detector at the LHC [1]. In addition to collecting data at the highest energies, which allow for probing the electroweak and symmetry breaking sector and searching for new heavy particles directly, exploring the energy frontier, the experiment also accumulates sizeable datasets at lower energies, exploring the luminosity frontier. In this regard, and in addition to joining ATLAS in the high- $p_{T}$ front, CMS complements the experiments dedicated to the study of $b$ hadrons, LHCb, and of heavy-ion collisions, ALICE. Owing to robust and flexible tracking, muon, and trigger systems and the ability to efficiently explore the highest LHC luminosities and the different collision systems, CMS is making significant and unique contributions to the heavy flavor (HF) realm. We report briefly on recent $\mathrm{HF}$ production results in $\mathrm{pp}, \mathrm{pPb}$ and $\mathrm{PbPb}$ collisions.

Hadronic systems involving heavy quarks, $b$ and $c$, provide a suitable laboratory in which to study Quantum Chromodynamics (QCD). Measurements of differential production cross sections have been performed for S-wave quarkonia [2] and $B^{+}$mesons [3] with initial Run2 data at $13 \mathrm{TeV}$, which extend similar measurements that had been performed at $7 \mathrm{TeV}$. The smaller pp dataset collected at $5 \mathrm{TeV}$ has allowed corresponding measurements of $J / \psi$ [4], $\psi(2 \mathrm{~S})$ [5], $D^{0}$ [6], $B^{+}$[7], $B_{s}^{0}$ [8] and $\Upsilon(n S)$ [9]. Similar results having been performed for the quarkonium states also at $2.76 \mathrm{TeV}$, these allow a study of the $\sqrt{s}$ dependence of the production cross sections. The heavier $B_{c}$ meson has also been probed [10]. Polarization measurements have been performed for the Swave quarkonium states at $7 \mathrm{TeV}$, and also for the $\Lambda_{b}$ baryon [11]. Associated production of heavy flavor with other states provide a complementary view that is also explored, e.g. double quarkonia $J / \psi+J / \psi[12]$ and $\Upsilon(1 S)+\Upsilon(1 S)[13], \psi+$ jets [14], and $W+$ charm [15].

The bottomonium system plays a special role for an improved understanding of underlying production mechanisms that has been extensively explored in Run1 [16]. With the Run2 data an higher $p_{T}$ region has been probed for S-wave states and enough yields also exist to study P-wave states. The $\chi_{b, J}(3 P)$ triplet $(J=0,1,2)$ is interesting in particular for allowing to infer how its properties are affected by the proximity to the open beauty $(B \bar{B})$ threshold. The merged states had been observed by ATLAS [17] earlier in Run1 (the first newly observed state at the LHC). Using an integrated luminosity of $80 \mathrm{fb}^{-1}$ collected during Run2, CMS has reported the observation of the resolved $\chi_{b, 1}(3 P)$ and $\chi_{b, 2}(3 P)$ states, along with a measurement of their masses [18]. Studies of P-wave $B_{s}^{0}$ mesons have been also pursued, leading to the observation of the $B_{s 2}^{*} \rightarrow B^{0} K_{S}$ and evidence of the $B_{s 1} \rightarrow B^{* 0} K_{S}$ decays [19].

Heavy quarks provide sensitive and novel hard probes of the hot QCD medium created in heavy ion collisions. Important properties of the medium such as its temperature and the mechanisms of energy loss and their flavor dependence are probed. These are inferred by comparing cross sections across various systems (in addition to $\mathrm{PbPb}$ and $\mathrm{pp}$, also $\mathrm{pPb}$ for quantifying so-called cold nuclear matter effects) as well as particle distributions and correlations. The exploration of the bottomonium family of states resulted in the flagship Run1 observation of sequential quarkonium suppression [20], which has more recently been confirmed at the higher Run2 energies [21]. The charmonium states have been similarly explored $[4,5]$, which in addition to melting (via Debye screening) are sensitive to recombination effects. In addition to quarkonia also open-flavor states are being actively investigated. These include the $D^{0}$ meson that is successfully reconstructed in its fully hadronic final state also in $\mathrm{PbPb}$ collisions [6]. B mesons are traditionally accessed in the heavy-ion environment in an inclusive fashion, via their leptonic decays, or more effectively via displaced charm, i.e. non-prompt $\psi$ [4] and $D$ [22]. In addition, however, CMS has directly 

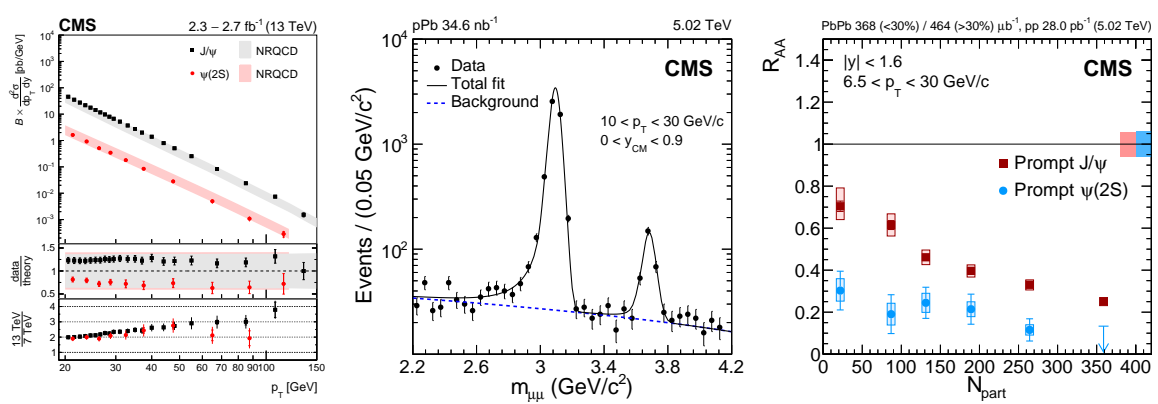

Figure 1: The charmonium system. Left: Differential cross section in pp collisions at $13 \mathrm{TeV}$ [2]. Middle: Reconstruction in pPb collisions at $5 \mathrm{TeV}$ [5]. Right: Suppression factor in $\mathrm{PbPb}$ collisions at $5 \mathrm{TeV}$ [4].
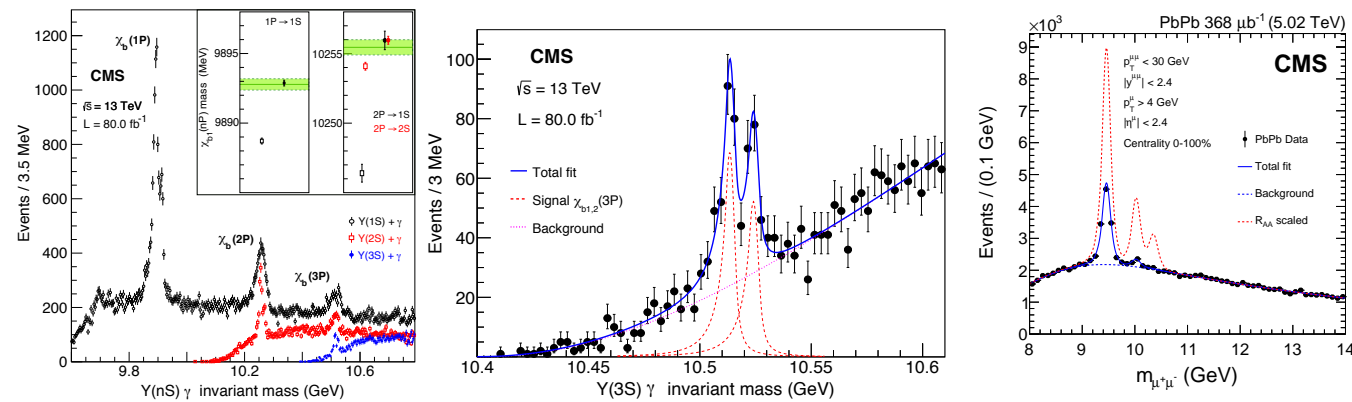

Figure 2: The bottomonium system. Left: The $\chi_{b}$ P-wave states, reconstructed in their decays to $\Upsilon \gamma$ [18]. Middle: Mass splitting of the $\chi_{b, 1}(3 P)$ and $\chi_{b, 2}(3 P)$ states, in the decays to $\Upsilon(3 S) \gamma$ [18]. Right: The $\Upsilon$ $\mathrm{S}$-wave states, in $\mathrm{PbPb}$ collisions (data points) and relative $\left(R_{\mathrm{AA}}\right.$-scaled) yields in pp collisions (dashed red line), at $5 \mathrm{TeV}$ [21]. The $\Upsilon(n S)$ and $\gamma$ particles are reconstructed resp. in the $\mu^{+} \mu^{+}$and $e^{+} e^{-}$final states.

reconstructed $B$ mesons for the first time in the ion collision environment $[23,7,8]$, which allows to distinguish the mesons' flavor. The measured suppression for various meson species is collected in Fig. 3 (right), which reflects the flavor/mass dependence of energy loss with expected hierarchy. The $B_{s}^{0}$ meson has been reconstructed in the various collision systems [8, 23] (Fig. 4), hinting at a potential strangeness enhancement (relative to the lighter species); although here as elsewhere additional $\mathrm{PbPb}$ data will be crucial. Additional ion data will be no less precious for exploring even heavier, rarer heavy flavor probes. These include among others also the top quark, which was already observed in $\mathrm{pPb}$ [24] paving the way for its future investigation in $\mathrm{PbPb}$ collisions.

All CMS public results may be accessed via the Collaboration's publication portal [25]. The full Run2 dataset shall allow for improved precision but also for the exploration of additional heavy flavor processes and sensitive probes of the strong and electroweak sectors of the standard model as well as potentially the physics that lies beyond.

\section{References}

[1] CMS Coll., JINST 3 (2008) S08004, doi:10.1088/1748-0221/3/08/S08004

[2] CMS Coll., PLB 780 (2018) 251, arXiv:1710.11002 [hep-ex]
[3] CMS Coll., PLB B 771 (2017) 435, arXiv:1609.00873 [hep-ex]

[4] CMS Coll., EPJC 78 (2018) 6, 509, arXiv:1712.08959 [nucl-ex]

[5] CMS Coll., submitted to PLB, arXiv:1805.02248 [hep-ex] 

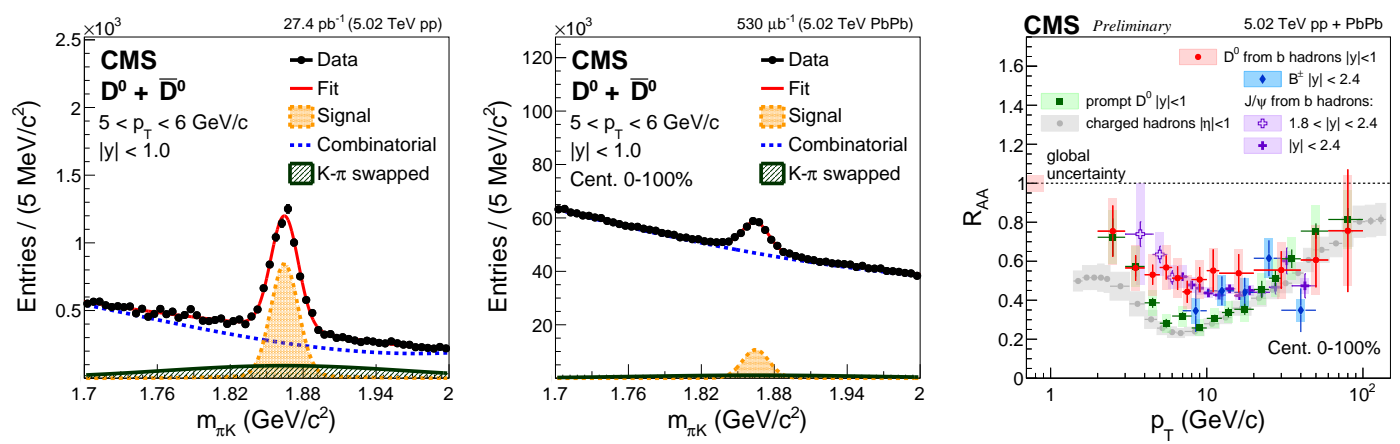

Figure 3: The $D^{0}$ system. Signal reconstructed in the $D^{0} \rightarrow K^{+} \pi^{-}$decay, in pp (Left) and $\mathrm{PbPb}$ (Middle) collisions at $5 \mathrm{TeV}$ [6]. Right: Nuclear modification factor as function of $p_{T}$ for various hadron species [22].
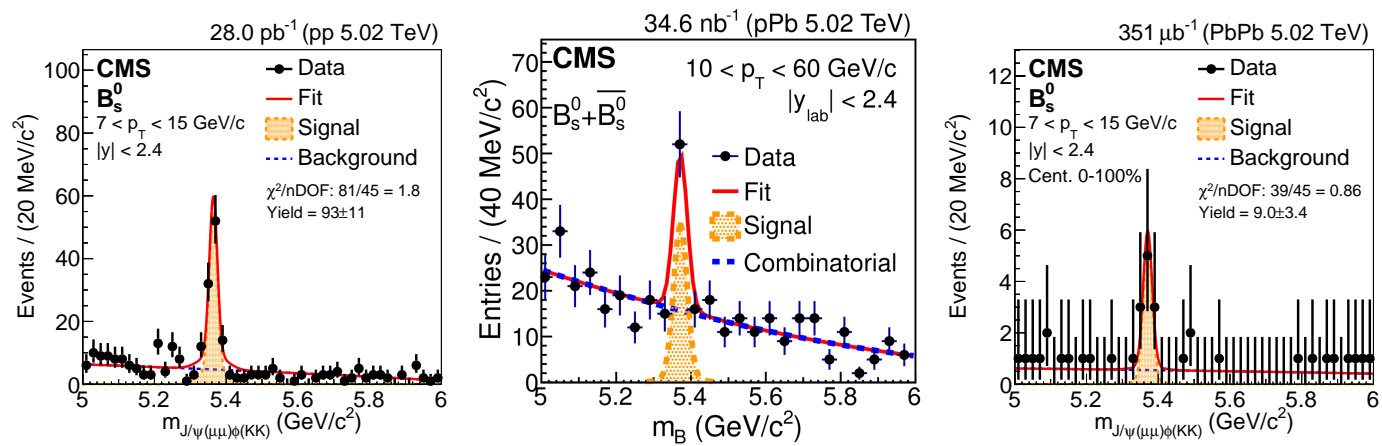

Figure 4: The $B_{s}^{0}$ system. The meson is reconstructed in the $B_{s}^{0} \rightarrow J / \psi \phi$ decay, respectively in pp, $\mathrm{pPb}$ and $\mathrm{PbPb}$ collisions at $5 \mathrm{TeV}[8,23]$. The $J / \psi$ and $\phi$ mesons are reconstructed resp. as $\mu^{+} \mu^{-}$and $K^{+} K^{-}$.

[6] CMS Coll., PLB 782 (2018) 474, arXiv:1708.04962 [Nucl-ex]

[7] CMS Coll., PRL 119 (2017) 15, 152301, arXiv:1705.04727 [hep-ex]

[8] CMS Coll., submitted to PLB, arXiv:1810.03022 [hep-ex]

[9] CMS Coll., submitted to PLB, arXiv:1805.09215 [hep-ex]

[10] CMS Coll., CMS-PAS-BPH-13-002 (2018), https://cds.cern.ch/record/2621286

[11] CMS Coll., PRD 97 (2018) 7, 072010 , arXiv:1802.04867 [hep-ex]

[12] CMS Collaboration, JHEP 1409 (2014) 094, arXiv:1406.0484 [hep-ex]

[13] CMS Collaboration, JHEP 1705 (2017) 013, arXiv:1610.07095 [hep-ex]

[14] CMS Coll., CMS-PAS-HIN-18-012 (2018), https://cds.cern.ch/record/2318344

[15] CMS Coll., CMS-PAS-SMP-17-014 (2018), https://cds.cern.ch/record/2314570
[16] Z.Hu, N.Leonardo et al, IJMP A 32 (2017) 19n20, 1730015, arXiv:1708.02913 [hep-ex]

[17] ATLAS Coll., PRL 108 (2012) 152001, arXiv:1112.5154 [hep-ex]

[18] CMS Coll., PRL 121 (2018) 092002, arXiv:1805.11192 [hep-ex]

[19] CMS Coll., submitted to EPJC, arXiv:1809.03578 [hep-ex]

[20] CMS Collaboration, PRL 109 (2012) 222301, arXiv:1208.2826 [nucl-ex]

[21] CMS Coll., PRL 120 (2018) 14, 142301, arXiv:1706.05984 [hep-ex]

[22] CMS Coll., submitted to PRL, arXiv:1810.11102 [hep-ex]

[23] CMS Coll., PRL 116 (2016) 3, 032301, arXiv:1508.06678 [nucl-ex]

[24] CMS Collaboration, PRL 119 (2017) 24, 242001, arXiv:1709.07411 [nucl-ex]

[25] CMS public results pages, http://cms-results.web.cern.ch/cmsresults/public-results/publications/ 